\title{
INDICADORES DE QUALIDADE NA CONDUÇÃO DE ESTUDOS CLÍNICOS
}

\section{QUALITY INDICATORS IN THE CONDUCT OF CLINICAL STUDIES}

\author{
Milena Artifon ${ }^{1}$, Adriele Scopel Guerra ${ }^{1}$, \\ Fabio Costa Jorge Gonçalves ${ }^{1}$, Rafael Leal Zimmer ${ }^{1}$, \\ Taiane Alves Vieira ${ }^{1}$, Vera Lorentz de Oliveira-Freitas ${ }^{1}$
}

\section{RESUMO}

Clin Biomed Res. 2019;39(4):346-352

1 Serviço de Gestão em Pesquisa, Grupo de Pesquisa e Pós-graduação (GPPG), Hospital de Clínicas de Porto Alegre (HCPA) Porto Alegre, RS, Brasil.

Autor Correspondente: Vera Lorentz de Oliveira-Freitas vlfreitas@hcpa.edu.br Hospital de Clínicas de Porto Alegre (HCPA)

Ramiro Barcelos, 2350

90035-903, Porto Alegre, RS, Brasil.
Introdução: A geração, análise e difusão de indicadores pertinentes é uma das estratégias fundamentais do processo de gestão de qualidade. Indicadores são mensurações que avaliam direta ou indiretamente os processos e desfechos da assistência ao participante de pesquisa. Padrões rigorosos de qualidade garantirão a validade dos dados obtidos nos estudos clínicos.

Métodos: Estudo transversal descritivo e analítico, com dados coletados no Centro de Pesquisa Clínica de um Hospital Universitário, durante Novembro/2016 a Fevereiro/2019 quanto ao tempo de inclusão de dados no electronic case report form (IID), tempo de comunicação de desvios de protocolo ao Comitê de Ética em Pesquisa (ICD), tempo de resposta aos feasibilities recebidos (IRF) e tempo de resposta às pendências de monitoria (IRP).

Resultados: Variações substanciais foram encontradas entre os escores de qualidade ao longo de 27 meses. O desempenho geral da equipe do Escritório de Projetos em Pesquisa alcançou a classificação excelente ou satisfatória em $61,40 \%$ das observações: 50,87\% e 10,52\% respectivamente. Entre 38,59\% de observações críticas, $17,54 \%$ foram expressas pelo IRP, seguido de $12,28 \%$ por ICD e $7 \%$ atribuído ao IID que não atingiram a meta proposta.

Conclusão: O Escritório de Projetos em Pesquisa elencou dois indicadores de qualidade, IID e IRP, que podem melhorar o tempo e a eficácia das entregas propostas da equipe.

Palavras-chave: Projetos de pesquisa; protocolos clínicos; avaliação da pesquisa em saúde; indicadores de projetos de pesquisa e desenvolvimento; integridade em pesquisa

\section{ABSTRACT}

Introduction: The generation, analysis and dissemination of relevant indicators is a fundamental strategy in the quality management process. Indicators are measurements that directly or indirectly evaluate the processes and outcomes of the care provided to research participants. Rigorous quality standards will guarantee the validity of the data obtained in clinical studies.

Methods: This descriptive, analytical cross-sectional study collected data at the Clinical Research Center of a University Hospital, covering the period from November 2016 to February 2019, regarding the time of data inclusion in the electronic case report form (IID), communication of protocol deviations to the Research Ethics Committee (ICD), response time to received feasibilities (IRF) and response time to monitoring pending issues (IRP). 
Results: Substantial variations were found between quality scores in a period of 27 months. The overall performance of the Research Project Office team was excellent or satisfactory in $61.40 \%$ of the observations $(50.87 \%$ and $10.52 \%$ respectively). Among critical observations (38.59\%), $17.54 \%$ were expressed by IRP, $12.28 \%$ by ICD and $7 \%$ by IID, indicating those that did not reach the proposed target.

Conclusions: The Research Project Office has listed two quality indicators, IID and IRP, which can improve the time and effectiveness of team deliveries.

Keywords: Research projects; clinical protocols; health research evaluation; research and development project indicators; research integrity

A preocupação com a qualidade e os temas que a cercam são pautas nas organizações para o aprimoramento de seus produtos e serviços ${ }^{1}$. Os altos custos e a necessidade de promover a equidade de acesso aos recursos têm direcionado os esforços no sentido de buscar evidências objetivas de que os serviços de saúde são providos de maneira eficiente, enquanto mantém e melhoram a qualidade do cuidado do paciente. Para isso, é necessário clareza do que se pretende medir, qualidade e precisão na produção de informações que comporão os indicadores, além de muita prudência na interpretação das informações disponíveis. A avaliação, ao final, permite formar um juízo de valor sobre o nível de qualidade alcançado, segundo critérios que envolvam a estrutura, o processo e o resultado. Aponta problemas e mostra a necessidade de buscar estratégias para correção de falhas ou para a melhoria de aspectos não satisfatórios. $\mathrm{O}$ desenvolvimento de intervenções e estratégias direcionadas para a melhoria dos indicadores de qualidade apresenta-se como importante ferramenta para o alcance de resultados positivos relacionados às condições de saúde, a gestão dos recursos e aos níveis de satisfação, tanto dos profissionais de saúde quanto dos usuários do sistema².

Os indicadores são utilizados como ferramentas de aferição da qualidade em saúde para monitorar e avaliar todo o processo que envolve a assistência ${ }^{3}$. Necessariamente, uma das características fundamentais dos indicadores é estabelecer um padrão normativo a partir do qual se avalia o estado social da realidade em que se quer intervir possibilitando descrever e comparar aspectos da realidade com dados anteriores através de conceitos e fórmulas. As áreas incluem: limites, configuração e métricas do processo, além de metas para melhoria do desempenho ${ }^{4}$. A geração, análise e difusão de indicadores pertinentes é uma das estratégias fundamentais do processo de gestão de qualidade assistencial ${ }^{5}$.

Assim como na assistência, existe a necessidade de avaliação da qualidade também no âmbito da pesquisa clínica. É inegável o fato de que, locais onde os estudos clínicos são realizados devem ter condições estruturais, equipe treinada e gerenciamento específico de forma a proteger os participantes de pesquisa ${ }^{6}$. Padrões rigorosos de qualidade, adesão ao protocolo, registro preciso de dados, atendimento aos critérios de inclusão e exclusão, além da comunicação contínua com o Comitê de Ética em Pesquisa (CEP), garantirão a qualidade e integridade dos dados obtidos nos estudos clínicos ${ }^{7}$. Sobretudo, em consonância com as boas práticas clínicas, a gestão da qualidade em centros de pesquisa é fundamental para medir desempenho e permitir a detecção precoce e periódica de problemas relacionados à execução de protocolos ${ }^{8}$.

Tendo em vista o desenvolvimento de instrumentos de gestão confiáveis, o objetivo deste trabalho foi desenvolver e analisar os indicadores para o controle de qualidade na condução de estudos clínicos no Hospital de Clínicas de Porto Alegre, avaliando o tempo de inclusão de dados no e-CRF (Eletronic Case Report Form), tempo de comunicação de desvios de protocolo ao CEP, tempo de resposta aos questionários de viabilidade de estudos (feasibilities) recebidos, e tempo de resposta às pendências de monitoria.

\section{MÉTODOS}

Estudo transversal, descritivo e analítico, utilizando dados secundários. Os dados compilados para a análise foram coletados no Escritório de Projetos do Grupo de Pesquisa e Pós-Graduação-GPPG no Centro de Pesquisa Clínica-CPC do Hospital de Clínicas de Porto Alegre-HCPA de novembro de 2016 a fevereiro de 2019. As variáveis dos estudos ativos foram observadas e agrupadas em quatro indicadores, sendo eles: tempo de inclusão de dados no formulário eletrônico de caso clínico, o e-CRF (IID), tempo de comunicação de desvios de protocolo ao Comitê de Ética em Pesquisa (ICD), tempo de resposta aos feasibilities recebidos (IRF), e tempo de resposta às pendências de monitoria (IRP).

O primeiro período do estudo compreendeu a construção dos indicadores conforme os aspectos 
normativos demonstrados na Tabela 1, e inicio da análise de desempenho das atividades. Seguindo a experiência clássica americana ${ }^{9}$, após nove meses de observações foi identificada a necessidade de limitar a configuração de alguns parâmetros do processo para avaliação da qualidade. Assim iniciou-se o segundo período do estudo incluindo rigor nos padrões de observação e uma tabela para a entrada dos dados denominada "quadro gerencial de indicadores", agora disponibilizada através da plataforma eletrônica adotada institucionalmente. A coleta de todos os dados foi realizada por assistente do escritório de projetos de pesquisa clínica do HCPA e com a participação de quatro cursistas do Programa Institucional de Curso e Capacitação Profissional (PICCAP) em Condução de Estudos Clínicos. $A$ validação da observação é feita anualmente através do treinamento dos observadores (assistentes de pesquisa). A concordância excelente entre os observadores deve ser superior a $80 \%$. Os dados calculados são analisados mensalmente em reunião do escritório de projetos/pesquisa clínica.

Tabela 1: Qualificação da natureza do impacto dos indicadores ${ }^{9}$.

Objetivos da política/ programa
Objetivos da avaliação

Avaliar processo
Tipo de pesquisa

Critérios de aferição
Atingir metas
Execução de metas propostas
Eficácia

\section{Indicador tempo de inclusão de dados no e-CRF (IID)}

O indicador IID mede o tempo (em dias) necessário para a inclusão de dados de participantes na ficha clínica eletrônica. Para o cálculo da média mensal, foi considerado o "número total de dias para a inclusão de cada relatório de dados dos participantes no e-CRF" (numerador) / "número total de relatórios inclusos" (denominador). O tempo "zero" (0) dias foi adotado quando o dado foi inserido no e-CRF no mesmo dia em que a visita do estudo foi realizada no centro. A meta para o indicador IID é $100 \%$ dos dados incluídos no e-CRF em até 4 dias após a visita do estudo.

\section{Indicador tempo de comunicação de desvios de protocolo ao CEP (ICD)}

$O$ indicador ICD mede o tempo (em dias) necessário para a comunicação de desvios de protocolo ao CEP. A média mensal do indicador é gerada através de: "número total de dias para a comunicação de cada desvio de protocolo ao CEP" (numerador) / "número total de desvios comunicados" (denominador). A meta para o indicador ICD é $100 \%$ dos desvios de protocolo comunicados ao CEP em até 3 dias após a identificação do desvio pela equipe ou representante do patrocinador.

\section{Indicador tempo de resposta aos feasibilities recebidos (IRF)}

$\mathrm{O}$ indicador IRF mede o tempo (em dias) necessário para resposta aos feasibilities recebidos. A média mensal do indicador é gerada através de: "número total de dias para resposta aos feasibilities recebidos" (numerador) / "número total de feasibilities recebidos" (denominador). Os dados são fornecidos pelos assistentes de pesquisa após as respostas aos feasibilities recebidos via e-mail. A meta para o indicador IRF é $100 \%$ dos feasibilities respondidos em até 2 dias após recebimento.

\section{Indicador tempo de resposta às pendências de monitoria (IRP)}

O indicador IRP mede o tempo (em dias) necessário para resposta às pendências de monitoria recebidas. A média mensal do indicador é gerada através de: "número total de dias para resposta às pendências de monitoria recebidas" (numerador) / "número total de pendências de monitoria recebidas" (denominador). O tempo "zero" (0) dias foi adotado quando a monitoria do estudo finalizou sem pendência. A meta para o indicador IRP é $100 \%$ das pendências respondidas em até 5 dias após a visita de monitoria.

\section{Caracterização dos conceitos dos indicadores}

Seu nível hierárquico é operacional, com perspectiva para os clientes (pesquisadores responsáveis e indústria farmacêutica). Os conceitos foram atribuídos aos indicadores, segundo critérios previamente estabelecidos em relação ao número de dias definido para a meta ser atingida caracterizada na tabela 2. Utilizaram-se as médias e desvios padrão ( $\pm D P$ ) para todas as análises do tempo de execução das atividades. O tempo foi calculado através da média dos dias entre a identificação da demanda até sua conclusão. 
Tabela 2: Referência dos conceitos atribuídos aos indicadores.

\begin{tabular}{|c|c|c|c|c|}
\hline \multirow[t]{2}{*}{ Indicadores } & \multicolumn{4}{|c|}{$\begin{array}{c}\text { Conceitos } \\
\text { (tempo em dias) }\end{array}$} \\
\hline & Excelente & Satisfatório & Insatisfatório & Crítico \\
\hline $\begin{array}{l}\text { Tempo de inclusão de dados no eCRF* do } \\
\text { estudo: IID }\end{array}$ & $<4$ & $4 \leq 7$ & $>7 \leq 10$ & $>10$ \\
\hline $\begin{array}{l}\text { Tempo de comunicação de desvios de protocolo } \\
\text { ao CEP: ICD }\end{array}$ & $<3$ & $3 \leq 7$ & $>7 \leq 10$ & $>10$ \\
\hline $\begin{array}{l}\text { Tempo de resposta aos feasibilities recebidos: } \\
\text { IRF }\end{array}$ & $<2$ & $2 \leq 7$ & $>7 \leq 10$ & $>10$ \\
\hline $\begin{array}{l}\text { Tempo de resposta às pendências de monitoria: } \\
\text { IRP }\end{array}$ & $<5$ & $5 \leq 7$ & $>7 \leq 10$ & $>10$ \\
\hline
\end{tabular}

\section{RESULTADOS}

Os escores de qualidade variaram substancialmente entre os períodos conforme demonstrado nos Gráficos 1 e 2. Entre Novembro de 2016 a Julho de 2017 os dados observados através dos indicadores IRP, ICD e IID, em sua maioria, encontram-se dentro da classificação esperada. O indicador IRF também foi acompanhado e algumas observações satisfatórias foram encontradas e uma insatisfatória durante 0 período avaliado. Entretanto, este resultado não representava a rotina do escritório de projetos naquele momento. Sendo assim, este indicador foi retirado da análise posterior.

Gráfico 1: Análise do período Novembro/2016-Julho/2017.

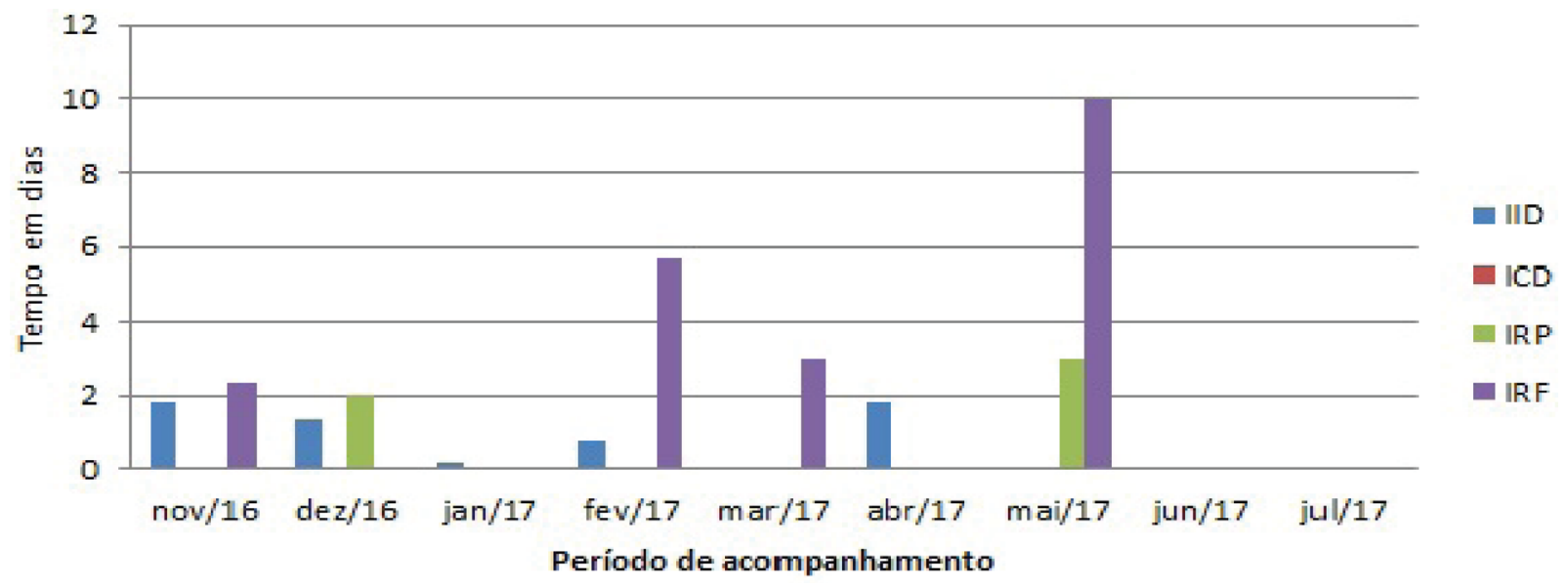

Gráfico 2: Análise do período Agosto/2017-Fevereiro/2019.

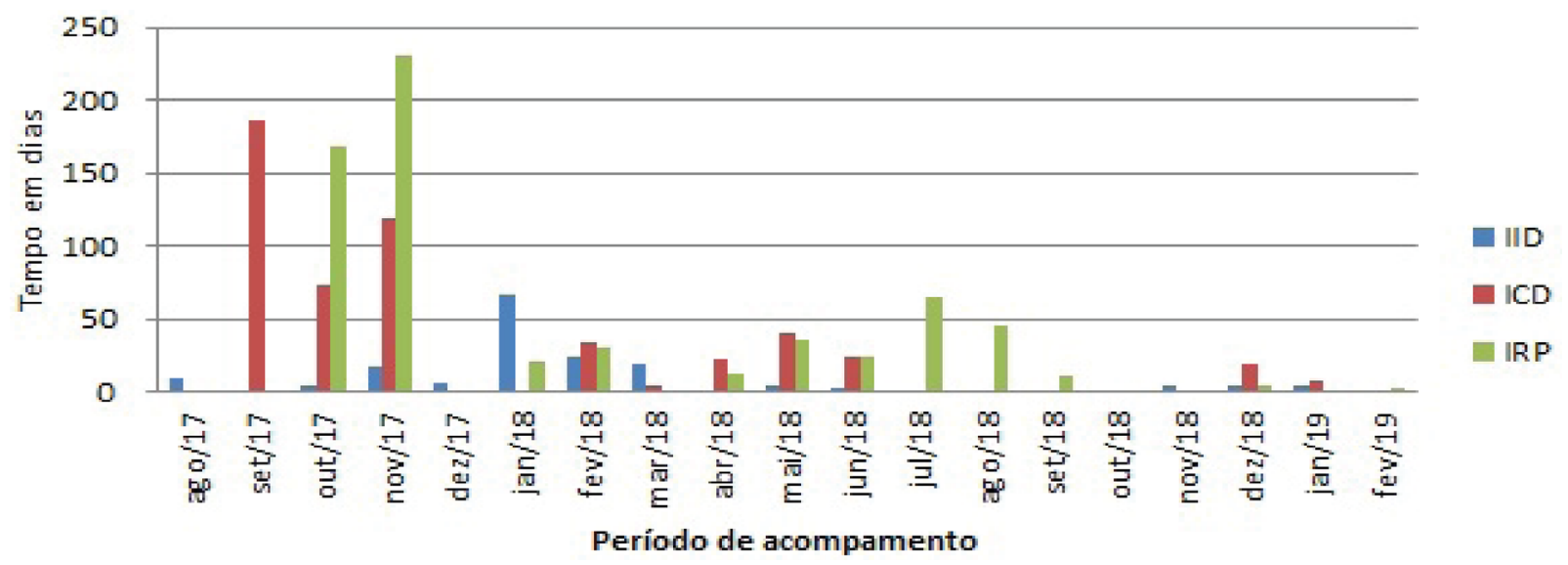


Artifon et al.

No segundo período de acompanhamento das atividades, entre agosto de 2017 a fevereiro de 2019, o desempenho geral da equipe do Escritório de Projetos alcançou a classificação excelente ou satisfatória em $61,40 \%$ das observações: $50,87 \%$ e $10,52 \%$ respectivamente (Gráfico 2). Foram detectadas $38,59 \%$ observações críticas, que foram expressas pelo IRP $(17,54 \%)$, seguido por ICD $(12,28 \%)$ e pelo IID $(7 \%)$ e não atingiram a meta proposta.

\section{DISCUSSÃO}

A avaliação da relação entre as metas propostas e as metas alcançadas não foi a principal observação do estudo quando comparado a constatação da necessidade de limitantes de aferição para análise de desempenho das atividades relativas a condução de estudos clínicos. Todos os indicadores obtiveram melhora em seus conceitos, sendo que os indicadores IID e IRP foram elencados como os mais representativos por concentrarem grande parte das atividades da equipe. A seleção dos indicadores contemplou, principalmente, os parâmetros de comparabilidade, disponibilidade e acessibilidade que proporcionasse as condições necessárias para o seguimento ao longo do tempo.

A primeira fase do estudo foi encerrada com a inclusão de novos limitantes nas datas de aferição dos indicadores, sendo eles, a data da randomização dos participantes adotada como baseline para o seguimento das visitas (IID), assim como a data da inclusão do primeiro participante definida como o início do acompanhamento dos estudos, e a data de recebimento do e-mail em seguimento a visita de monitoria como o início formal de uma pendência de monitoria no IRP. Visto que a qualidade dos dados gerados desempenha um importante papel no resultado do estudo ${ }^{10}$, o IID foi o primeiro alvo - o menor tempo para inclusão dos dados possibilita mais rápida verificação e identificação de dados faltantes, além de minimizar as chances de perda de dados por lapso de preenchimento ${ }^{11}$. Por conseguinte, determinou-se o IRP, onde o menor tempo de resposta às pendências recebidas pós monitorização dos dados reflete positivamente no grau de satisfação dos clientes. O contratante da prestação de serviço precisa de dados legítimos, consistentes e acima de tudo contemporâneos.

Os dados com classificação crítica, em sua maioria, encontram-se nas observações do indicador IRP. Verificou-se que os atrasos são decorrentes da necessidade de verificação em documento-fonte durante as visitas de monitoria, porém não representa o tempo que a pendência foi efetivamente solucionada pela equipe do EP. Neste sentido, sugeriu-se que o parâmetro de coleta fosse alterado, contabilizando o tempo que a equipe de pesquisa obteve a resolução, e não a data atribuída pelo monitor do estudo. Dessa forma, esse indicador, efetivamente, reflete a qualidade e a produtividade da equipe.

A proposta de incluir o ICD foi sustentada pela necessidade da contemporaneidade das notificações de desvios de protocolo ao CEP. O menor tempo para a comunicação de desvios possibilita a detecção de pontos críticos. A norma oficial brasileira abre espaço a estratégias de monitoramento aos centros com o objetivo de auxiliar os pesquisadores na análise de riscos e benefícios durante o desenvolvimento de projetos de pesquisa ${ }^{12}$. As diretrizes internacionais apontam a importância da utilização de mecanismos, ferramentas e instrumentos próprios de inter-relação, visando a proteção dos participantes de pesquisa de forma coordenada e descentralizada ${ }^{13}$. A rápida notificação permite ao CEP, quando necessário, alertar os responsáveis para possíveis providências, no sentido de mitigar falhas, junto a equipe de pesquisa. Sobretudo, esse sistema de monitoramento interno pode colaborar para a reavaliação de métodos e a proposição de medidas preventivas ${ }^{14}$. Atualmente, a equipe do EP segue um fluxo de notificação de desvios de protocolo com as seguintes etapas: 1) identificação do DP pela equipe ou monitor; 2) narrativa da ocorrência para ciência do Pesquisador Responsável (PI); 3) notificação do desvio no Strategic Advisor (SA), sistema de padronização documental do HCPA. Incluindo em anexo a carta narrativa assinada pelo $\mathrm{PI}$; 4) retorno da avaliação do desvio realizada pela Unidade de Assuntos Regulatórios (UARP/GPPG/HCPA) enviado por e-mail e posterior arquivamento do estudo. Verificou-se um componente de aferição dependente de equipe externa ao EP, assim, o indicador não representa o desempenho desta unidade exclusivamente.

O menor tempo de resposta aos feasibilities recebidos demonstra alto grau de interesse institucional em captar novos estudos assim como a capacidade organizacional da equipe, que é fundamental para a condução de estudos. O IRF foi retirado da análise posteriormente, pois os protocolos conduzidos foram contratados após a etapa de verificação da viabilidade do estudo, respondidos pelos pesquisadores responsáveis. Assim, este indicador pode ser uma importante ferramenta de gestão se adotada quando os questionários forem direcionados para o EP.

A responsabilização nas atividades científicas e a confiança pública nos resultados de pesquisa são hoje percebidas como parte dos desafios éticos mais atuais na ciência contemporânea, sobretudo num momento em que se intensificam os diálogos entre ciência e sociedade ${ }^{15}$. A percepção de que uma contribuição significativa para a ciência é inseparável da responsabilização nas atividades de pesquisa se reflete em mudanças significativas nos critérios de julgamento sobre a qualidade e solidez de projetos 
e de artigos científicos ${ }^{16}$. Qualidade e solidez são características interligadas, que dependem da confiabilidade dos dados e de sua fiel representação nos registros de pesquisa, que podem ser afetadas por conflitos de interesses não revelados, que podem distorcer os registros de pesquisa e enganar a comunidade de pesquisa ${ }^{17}$. Novas propostas de indicadores poderiam incluir o conflito de interesses em pesquisa científica e acadêmica - que ocorre quando motivos alheios interferem na consecução de objetivos diretamente visados por uma instituição e se traduz em impeditivo ético para as pesquisas. Todo e qualquer conflito de interesses deve ser evitado a priori, e, quando verificado posteriormente, deve ser equacionado e solucionado imediatamente ${ }^{18}$. No Brasil, crescentes incentivos para o desenvolvimento de projetos colaborativos, em um ambiente internacional cada vez mais competitivo, podem gerar resultados ainda mais promissores se as instituições, periódicos acadêmicos, sociedades científicas e agências de financiamento estabelecerem medidas estratégicas para promover e sustentar a responsabilização nas atividades de pesquisa e a confiança pública na ciência ${ }^{15-17}$.

\section{Limitações}

O estudo realizado apresentou limitações em sua metodologia e análises, pode ter ocorrido um viés de aferição atribuído à forma de tabulação dos dados entre as fases analisadas. Não foi adotado um plano de ação com medidas preventivas e/ou corretivas quando os indicadores atingissem critérios insatisfatórios ou críticos, sendo uma proposta de melhoria para este controle.

\section{Conclusão}

Em comparação ao período inicial das coletas, todos os indicadores obtiveram melhora em seus conceitos, demonstrando assim que, com o seu monitoramento é possível melhorar os prazos das entregas, aumentando a efetividade nos serviços prestados pelo escritório de projetos ${ }^{14,19}$. Os dados evidenciaram a necessidade de elencar critérios rigorosos de padronização para geração e documentação dos dados. Neste contexto, o indicador IID e IRP foram os mais representativos para refletir o resultado das atividades em equipe. Assim, sua tabulação seguiu de forma prospectiva desde Fevereiro/2019.

\section{Agradecimentos}

O estudo contou com o apoio do Professor Eduardo Pandolfi Passos, Coordenador do Grupo de Pesquisa e Pós-graduação (GPPG) entre 2013 a 2015 e com a colaboração da Assessora do GPPG Rosane Schlatter, durante a transição de Coordenação do GPP, quando assumiu a Professora Patrícia Prolla. A participação da cursista Laura Pozzobom do PICCAP em Condução de Estudos Clínicos durante a coleta de dados na fase inicial.

\section{REFERÊNCIAS}

1. Brandão E, Oliveira P, Melo PFAS, Soares HPS, Araújo PSR, AguiarSantos AM, et. al. Utilização de indicadores da qualidade no ambulatório do Serviço de Referência Nacional em Filarioses. Rev A creditação. 2015;5(10):1-12.

2. Ferreira JM, Kulbok P, Silva CAB, Andrade FB, Costa ICC. Indicadores de qualidade na atenção primária à saúde no Brasil: uma revisão integrativa. Rev Cienc Plur. 2018;3(3):45-68.

3. Oliveira LS, Costa DN, Oliveira DML, Almeida HOC, Mendonça IO. Indicadores de qualidade nos serviços de urgência hospitalar. Cienc Biol Saude Unit. 2018;4(3):173-88.

4. Project Management Institute, Global Standard. Um Guia do conhecimento em gerenciamento de projetos - (Guia Pmbok®). $5^{\text {a }}$ ed. Newtown Square; 2014.
5. Klück MM, Silveira IP, Malvezzi ML, Ferreira J. Gestão de qualidade assistencial no HCPA: o papel dos Indicadores. 7th Qualihosp; 2007 Abr 3-Abr 4; Porto Alegre, RS. Porto Alegre: UFRGS; 2007 Abr. 1 p. (Resumos do Qualihosp).

6. Knatterud GL, Rockhold F, George SL, Barton FB, Davis CE, Fairweather $W R$, et al. Guidelines for quality assurance in multicenter trials: a position paper. Control Clin Trials. 1998 Oct;19(5):477-93.

7. Lousana G, Accetturi C. Gestão de um centro de pesquisa como fator de sucesso. Rio de Janeiro: Revinter, 2007.

8. International Conference on Harmonization of Technical Requirements for Registration of Pharmaceuticals for Human Use. ICH Harmonized Guideline. Integrated addendum to ICH E6(R1): guideline for good clinical practice E6(R2) [Internet]. 2015 [acesso 2 nov 2016]. Disponível: http://bit.ly/1L4YIA2.

9. Kayano J, Caldas EL. Indicadores para o diálogo. São Paulo: GT Indicadores; 2002.

10. Krishnankutty B, Bellary S, Kumar NB, Moodahadu LS. Data management in clinical research: an overview. Indian J Pharmacol. 2012;44(2):168-72.

11. Mayer, AG. Indicadores para gestão da pesquisa clínica: primeiras anotações. Porto Alegre: Fiocruz; 2008.

12. Conselho Nacional de Saúde (BR). Resolução CNS nº 466, de 12 de dezembro de 2012: aprova diretrizes e normas regulamentadoras de pesquisas envolvendo seres humanos. Diário Oficial da União [Internet]. 2013 Dez 12 [citado 31 março de 2019]. Disponível em: http://conselho.saude.gov.br/ resolucoes/2012/Reso466.pdf. 
13. Jalgaonkar SV, Bhide SS, Tripathi RK, Shetty YC, Marathe PA, Katkar J, Thatte UM. An Audit of protocol deviations submitted to an institutional ethics committee of a tertiary care hospital. PLoS One. 2016 Jan 6;11(1):e0146334.

14. FDA [internet]. Washington (DC); 2008. Compliance program guidance manual. [citado 31 Mar 2019]. Disponível em: http://www.fda.gov/downloads/ ICECI/EnforcementActions/ BioresearchMonitoring/ucm133773.pdf
15. Código de boas práticas científicas [Internet]. São Paulo: Fapesp. 2011 [citado 2020 Fev 5]. Disponível em: http://www.fapesp.br/boaspraticas/ codigo_050911.pdf

16. CNPq [Internet]. Brasília (DF). 2010 [citado 2020 Fev 5]. Diretrizes; [aproximadamente 2 telas]. Disponível em: http://www.cnpq.br/web/guest/ diretrizes

17. Cooperation between research institutions and journals on research integrity cases: guidance from the Committee on Publication Ethics (COPE) [Internet]. Londres: Cope.
2012 [citado 2020 Fev 5]. Disponível em: http://publicationethics.org/files/ Research_institutions_guidelines_ final.pdf

18. Pontifícia Universidade Católica do Rio Grande do Sul. Resolução $n^{\circ}$ 04/2006. Reitoria [Internet]. 2006 [citado 2020 Fev 5];1-3.

19. NIH IRB Professional Administrators Committee. Bethesda; 2005. Protocol Deviations and Violations; [citado 2019 Mar 31]. Disponível em: https:// www.genome.gov/Pages/Research/ Intramural/IRB/Deviation_Violation_ examples8-07.pdf

Recebido: 14 out, 2019 Aceito: 12 dez, 2019 